\title{
Clinical Activity of Selitrectinib in a Patient With Mammary Analogue Secretory Carcinoma of the Parotid Gland With Secondary Resistance to Entrectinib
}

\author{
Vaia Florou, MD¹; Christopher Nevala-Plagemann, MD; Jonathan Whisenant, MD¹; Patricia Maeda, MD²; \\ Glynn W. Gilcrease, MD¹; and Ignacio Garrido-Laguna, MD, PhD ${ }^{1}$
}

\begin{abstract}
NTRK gene fusions are found in $<1 \%$ of all cancers but are uniformly present in mammary analog secretory carcinomas (MASC) of the salivary glands. Two selective histology-agnostic tropomyosin receptor kinase (TRK) inhibitors are currently approved for malignancies with these oncogenic fusions. Resistance to TRK inhibition has been recognized, and the mediating mechanisms are presently being studied. This report describes a patient diagnosed with an MASC of the parotid gland who after undergoing multiple lines of treatment was found to have an ETV6-NTRK3 fusion and initiated TRK-targeted therapy using entrectinib. Upon disease progression, we performed tumor genetic sequencing that showed a secondary resistance mutation. The patient subsequently responded to selitrectinib, a nextgeneration TRK inhibitor.
\end{abstract}

J Natl Compr Canc Netw 2021;19(5):478-482 doi: $10.6004 /$ jnccn.2021.7022
${ }^{1}$ Division of Oncology, Department of Internal Medicine, Huntsman Cancer Institute, University of Utah School of Medicine, Salt Lake City, Utah; and ${ }^{2}$ Bayer Healthcare Pharmaceuticals, Whippany, New Jersey.
The mammary analog secretory carcinomas (MASCs) of the salivary gland were first described in 2010 by Skálová et al. ${ }^{1}$ The nomenclature of MASC was proposed because of the histologic and immunohistochemical similarities with the secretory carcinomas of the breast. They share a lobulated pattern; express MUC1, MUC4, and mammaglobin $^{1}$; and are stained positive with periodic acid-Schiff and mucicarmine. In addition to morphology, a pathognomonic characteristic of MASC is the presence of the ETV6-NTRK3 fusion, which is found in most patients with MASC reported thus far., ${ }^{1,2}$ Although the term "MASC" continues to be widely used in the literature, the fourth edition of the WHO Classification of Head and Neck Tumors designated this entity as a secretory carcinoma in an effort to standardize the nomenclature across different organs. ${ }^{3}$ Typically, secretory carcinomas are indolent, such as acinar cell carcinomas; however, high-grade transformation can occur, leading to accelerated progression and poor outcomes. ${ }^{4}$

The neurotrophic tropomyosin receptor kinases (NTRKs) are a family of 3 kinases (TRKA, TRKB, and TRKC) that play a pivotal role in neural development and are encoded by the NTRK1, NTRK2, and NTRK3 genes, respectively. ${ }^{5}$ NTRK mutations and TRK protein overexpression have been described in a variety of different tumor types, including colorectal, lung, melanoma, and acute myeloid leukemias. ${ }^{6}$ The most common mechanism for oncogenic TRK activation ${ }^{7}$ results from fusions involving the 3 NTRK genes: intra- or interchromosomal rearrangements form hybrid genes in which $3^{\prime}$ sequences of NTRK1, NTRK2, or NTRK3 that include the kinase domain are juxtaposed to $5^{\prime}$ sequences of a different gene. ${ }^{6}$ Although the TRK fusion proteins lead to full downstream signaling similar to fulllength TRK proteins, the predominant activated pathway may depend on the histology. ${ }^{6}$

Although the overall incidence of NTRK fusions in adult and pediatric tumors is extremely low $(<1 \%)$, they are found very commonly in some rare tumors, such as infantile fibrosarcoma $(86 \%-91 \%)$, secretory breast 
carcinoma (92\%), and MASC (93\%-100\%). ${ }^{8}$ Other NTRK abnormalities, such as mutations or amplifications, can affect up to $14 \%$ of patients with cancer, but whether these alterations are relevant in the oncogenic process or sensitive to TRK inhibition is unclear. ${ }^{8}$

Multiple nonspecific multikinase inhibitors have some activity against TRK fusion proteins, but there are currently 2 TRK inhibitors approved by the FDA for any solid tumor with NTRK gene fusions. Larotrectinib was the first tissueagnostic targeted agent approved in 2018 based on 3 multicenter, single-arm trials: LOXO-TRK-14001 (ClinicalTrials. gov identifier: NCT02122913), SCOUT (NCT02637687), and NAVIGATE (NCT02576431). A recently published pooled analysis of 159 patients with solid tumors treated in these clinical trials showed that the overall response rate (ORR) of 153 evaluable patients was $79 \%$ according to investigator assessment, with a median duration of response of 35.2 months. ${ }^{9}$ Overall, 21 patients with salivary gland tumors were included across these 3 studies. Despite high responses to larotrectinib, primary and acquired resistance were noted in a small number of patients. On-target resistance can occur in the TRK kinase domain via mutations in the front position (NTRK1 G595R or NTRK3 G623R), gatekeeper position (NTRK1 F589L), or kinase activation loop xDFG position (NTRK1 G667S or NTRK3 G696A). ${ }^{10}$

Entrectinib, a multikinase inhibitor that targets TRK, ROS1, and ALK, was approved 1 year after larotrectinib based on the cumulative data of 3 multicenter, single-arm clinical trials: ALKA-372-001 (EudraCT number: 2012-000148-88), STARTRK-1 (ClinicalTrials.gov identifier: NCT02097810), and STARTRK-2 (NCT02568267). A total of 54 patients were included in these trials with an ORR of $57 \%$ and a median duration of response of 6 months. ${ }^{11}$ The ORR in 7 patients with MASC who were treated using entrectinib was $86 \%$. Similar to larotrectinib, resistance to entrectinib was also mediated by secondary mutations - in 1 patient with colorectal cancer, 2 new mutations on NTRK1 (Gly595Arg, Gly667Cys) were found to confer resistance. ${ }^{11}$

Next-generation TRK inhibitors are currently in development and can overcome this acquired resistance to first-generation inhibitors. Selitrectinib (BAY 2731954) is a selective TRK inhibitor for which there is already evidence of clinical activity in tumors with acquired resistance mutations. Results of 31 patients treated in a phase I study (ClinicalTrials.gov identifier: NCT03215511) and in the expanded-access protocol for that study were recently presented and included patients previously treated with firstgeneration TRK inhibitors. ${ }^{12}$ The ORR was $34 \%$ among all evaluable patients and $45 \%$ for patients with confirmed on-target resistance mutations. The dose-expansion phase II trial of selitrectinib is currently ongoing (NCT03215511). A phase I/II trial of repotrectinib, another novel TRK inhibitor with preclinical evidence of overcoming resistance, is also ongoing (NCT04094610).
This report presents a patient with an MASC of the parotid gland who was treated using TRK inhibitors after numerous lines of prior systemic therapies and courses of radiation.

\section{Case Description}

A male patient aged 55 years was diagnosed initially with adenocarcinoma of unknown primary when he presented with a right mandibular mass. He underwent resection and radiation at the time of his diagnosis, but 3 years later he developed metastatic disease in the thoracic spine. He received radiation followed by stereotactic radiosurgery 1 year later for an adjacent lesion outside the prior radiation field. Approximately 6 months after stereotactic radiosurgery, the patient was found to have diffuse osseous metastatic disease, prompting initiation of systemic cytotoxic chemotherapy using carboplatin and paclitaxel. After 6 cycles of systemic therapy, repeat imaging revealed stable disease. One year later, the patient was noted to have oligoprogressive disease in his lower spine and pelvis, for which he received palliative radiation. Three months later, he was found to have multifocal progression of his metastatic osseous disease, for which gemcitabine was initiated. Due to disease progression, after 3 cycles the chemotherapy was changed to capecitabine, on which the patient remained for 2 years until eventually progressing.

Additional tissue studies at the time of progression, 8 years after the patient's original diagnosis, revealed androgen receptor expression by immunohistochemistry. He was started on androgen deprivation therapy and remained stable for 3 years before eventually developing further progressive disease to the lumbar spine requiring palliative radiation for pain control. Approximately 1 year later, he developed diplopia secondary to an osseous metastatic focus involving the orbit, which improved with radiation.

Around this time, next-generation sequencing of the tumor revealed an ETV6-NTRK3 fusion. His diagnosis was changed to MASC of the salivary gland, and the patient was subsequently treated with entrectinib in the STARTRK-2 trial. He completed 25 cycles of entrectinib with a partial response on imaging $(76 \%$ decrease in tumor burden). During this treatment, the patient developed grade 1 dysgeusia and dizziness, along with grade 3 fatigue and autonomic neuropathy. After nearly 2 years on treatment, he developed new osseous lesions and was taken off trial when he developed a pathologic fracture in his right humerus.

While the patient was off entrectinib, he rapidly developed symptomatic osseous disease involving axial and appendicular sites and the left calvarium with associated leptomeningeal involvement. The patient became severely debilitated from his disease burden and was admitted 
to the hospital with hypoxemic respiratory failure. Tissue obtained at that time revealed a new NTRK3 G623R mutation. In light of this mutation, the patient was treated with selitrectinib under an expanded access program due to his performance status decline. After initiation of selitrectinib, he experienced rapid symptomatic improvement within a few days and radiographic response of the leptomeningeal involvement on subsequent imaging (Figure 1). In addition, his osseous disease remained stable for approximately 8 months. He tolerated selitrectinib well, with only grade 1 nausea attributed to the investigational agent. Unfortunately, the patient developed multiple infectious complications related to chronic decubitus ulcers and eventually died 10 months after initiation of selitrectinib.

\section{Discussion}

Since the first oncogenic fusion involving the Philadelphia chromosome was described in 1960, driver fusions have been at the forefront of research in multiple diseases, including prostate adenocarcinoma, thyroid cancer, Ewing sarcoma, and other rare malignancies. For some malignancies, these driver fusions are pathognomonic and have diagnostic significance where histology may be inconclusive. Despite the growing number of oncogenic driver fusions that have been identified, only a few of them are targetable by currently approved therapies. For rare malignancies such as MASC, for which treatment options are limited, the identification of targetable genomic alterations such as those involving the NTRK genes is critical. The case described here highlights the utility of contemporary precision medicine and shows an example of targeted therapy leading to durable disease control of $>30$ months in a patient who had exhausted all other treatments.

The solvent front NTRK3 G623R mutation identified in this patient after treatment using entrectinib has been described in the literature as a mechanism of acquired resistance to first-generation TRK inhibitors. ${ }^{10,13,14}$ Ours is the first report of clinical activity of a second-generation TRK inhibitor in the setting of emergent resistance to a first-generation TRK inhibitor via the NTRK G623R mutation. Notably, this case report also shows the activity of the next-generation TRK inhibitor selitrectinib in a patient with MASC and central nervous system (CNS) involvement. The activity of the 2 approved TRK inhibitors (larotrectinib and entrectinib) in CNS disease has been described. In the entrectinib analysis from 3 clinical trials, 12 patients had CNS involvement at diagnosis, and the response rate was similar to that of those patients without CNS disease $(n=42): 50 \%$ and $60 \%$, respectively. ${ }^{11}$ Similarly, an analysis of 14 patients with either metastatic disease to the CNS $(n=5)$ or primary CNS tumors $(n=9)$ who received larotrectinib in 2 clinical trials ${ }^{15}$ showed response

\begin{tabular}{|c|c|c|c|}
\hline Baseline & BOR on entrectinib & $\begin{array}{l}\text { Progression of disease on } \\
\text { entrectinib }\end{array}$ & $\begin{array}{l}\text { Response to selitrectinib } \\
\text { (LOXO-195) }\end{array}$ \\
\hline $\begin{array}{l}\text { ETV6-NTRK3 } \\
\downarrow\end{array}$ & & $\begin{array}{ll}\text { PoD } & \text { NTRK3 } \\
& \downarrow \\
& \text { Selitrectinib }\end{array}$ & 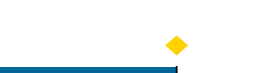 \\
\hline 0 & & 25 & 41 \\
\hline \multicolumn{4}{|c|}{ Genomic alterations } \\
\hline \multicolumn{2}{|c|}{$\begin{array}{l}\text { ETV6-NTRK3 fusion } \\
\text { NF1 Q589* }\end{array}$} & \multicolumn{2}{|c|}{$\begin{array}{l}\text { NTRK3 G623R, ETV6-NTRK3 fusion } \\
\text { NF1 Q598* } \\
\text { DNMT3A R8882C - subclonal }\end{array}$} \\
\hline \multicolumn{4}{|c|}{ Variants of unknown significance } \\
\hline \multicolumn{2}{|c|}{ STAT4 (A118T), ZNF703 (K300R) } & \multicolumn{2}{|c|}{$\begin{array}{l}\text { ACTB }\left(\mathrm{K} 118^{\star}\right), \text { BCL11B (R855H), HDAC7 (R166H), } \\
\text { MYO18A (R1351H), NCOR2 (K1819M), STAT3 (A376T), } \\
\text { STAT4 (A118T), ZNF703 (K300R), ZRSR2 (G438del) }\end{array}$} \\
\hline
\end{tabular}

Figure 1. Upper row: most characteristic imaging studies showing response to therapy with improvement in the dural thickening and leptomeningeal enhancement. Lower rows: tumor genetic sequencing findings at 2 different timepoints; prior entrectinib on the left and on PoD on entrectinib on right.

Abbreviations: BOR, best overall response; PoD, progression of disease. 
rates of $60 \%$ and $11 \%$, respectively. In the pooled analysis of 159 patients treated with larotrectinib, 13 had known brain metastases before enrollment, although baseline brain imaging was not required. ${ }^{9}$ Among them, 9 of 12 (75\%) experienced a response, including all sites of disease. Patients with primary CNS tumors were excluded from the analysis.

Recent work has shown that the molecular spectrum of MASC is even broader than previously thought. In addition to NTRK3, which is involved in most secretory carcinomas of salivary glands, other genes such as $A L K$, $M E T$, and RET have also been implicated in the pathogenesis of this rare disease ${ }^{16}$ For some of these genetic aberrations, targeted therapies are already FDA-approved for different indications. Selpercatinib (an RET inhibitor) is approved for RET fusion-positive non-small cell lung cancer, thyroid cancer, and RET-mutant medullary thyroid cancer. ${ }^{17-19}$ Capmatinib (an MET kinase inhibitor) was recently approved for patients with non-small cell lung cancer with mutations that led to MET exon 14 skipping. The role of these small molecules in patients with MASC harboring $A L K, M E T$, or RET aberrations will need to be evaluated in the future.

The next step in TRK inhibition lies in mechanistically exploring the pathways of acquired resistance to unveil ways to overcome them. Next-generation TRK inhibitors have shown activity against solvent front mutations. Both of the next-generation TRK inhibitors currently in clinical studies (selitrectinib and repotrectinib) maintain activity in solvent front mutations, gatekeeper mutations, and compound mutations. ${ }^{20}$

In addition, there is preclinical and clinical evidence of off-target resistance via activation of the downstream mitogen-activated protein kinase (MAPK) pathway. ${ }^{21} \mathrm{Si}-$ lencing of BIM, downstream of the MAPK pathway, contributed to resistance to entrectinib in 2 new TPR-TRKA driven mice models in pancreatic and lung cancer. ${ }^{22}$ In these models, vertical inhibition using entrectinib + cobimetinib (an MEK inhibitor) delayed the onset of resistance to entrectinib. Thus, upfront dual targeting of the implicated pathways may delay the emergence of resistant tumor clones and lead to more durable disease control compared with monotherapy.

Another example of off-target mechanisms of resistance on second-generation TRK inhibitors has been described in a patient with a gastrointestinal stromal tumor harboring a TPM3-NTRK1 fusion. ${ }^{23}$ After treatment with selitrectinib, activation of KRAS signaling via a gain-of-function KRAS G12V mutation was identified. ${ }^{23}$ Furthermore, compared with when the patient was treated using larotrectinib, more robust immune and inflammatory signatures were found on progression to selitrectinib. ${ }^{23}$ In addition, there was a higher density of CD8-positive $\mathrm{T}$ cells and macrophages in posttreatment tumor biopsies, suggesting a potential role of combination approaches using TRK inhibitors and immune checkpoint inhibition.

\section{Conclusions}

Moving forward, treatment strategies testing TRK inhibitors in combination with checkpoint or MAPK pathway inhibitors are warranted. Combination approaches are likely needed to delay the emergence of resistance, given that responses to single-agent targeted therapy are often short-lasting. One of the first examples of such a paradigm is in melanoma, for which BRAF combined with MEK inhibition targeting the MAPK reactivation is superior to BRAF inhibition alone. ${ }^{24}$ In contrast, in BRAF-mutated colon cancer, resistance to BRAF inhibition is mediated via epidermal growth factor receptor (EGFR). ${ }^{25}$ This process was shown in a phase III clinical trial in previously treated patients with BRAF-mutated colorectal cancer; BRAF with EGFR inhibition with or without MEK inhibition was superior to the standard-of-care cytotoxic chemotherapy used in this disease. ${ }^{26,27}$ Lastly, the I-PREDICT trial showed the feasibility and safety of combining targeted therapies following molecular tumor board evaluation of individual patient tumor genomic profiles. ${ }^{28}$ The study showed that a multitarget approach improved disease control and survival rates, compared with when fewer genetic alterations were targeted. $^{28}$

Submitted November 1, 2020; accepted for publication February 1, 2021.

Disclosures: Dr. Maeda has disclosed being employed by and owning stock in Bayer Healthcare Pharmaceuticals. Dr. Garrido-Laguna has disclosed receiving grant/research support from Bayer/Loxo Oncology. The remaining authors have disclosed that they have not received any financial consideration from any person or organization to support the preparation, analysis, results, or discussion of this article.

Funding: Research reported in this article was supported by the Foundation for the NIH under award number P30CA042014.

Disclaimer: The content is solely the responsibility of the authors and does not necessarily represent the official views of the $\mathrm{NIH}$.

Correspondence: Vaia Florou, MD, Division of Oncology, Department of Internal Medicine, Huntsman Cancer Institute, University of Utah School of Medicine, 2000 Circle of Hope, Salt Lake City, UT 84112.

Email: vaia.florou@hci.utah.edu

\section{References}

1. Skálová A, Vanecek T, Sima R, et al. Mammary analogue secretory carcinoma of salivary glands, containing the ETV6-NTRK3 fusion gene: a hitherto undescribed salivary gland tumor entity. Am J Surg Pathol 2010;34: 599-608.

2. Majewska H, Skálová A, Stodulski D, et al. Mammary analogue secretory carcinoma of salivary glands: a new entity associated with ETV6 gene rearrangement. Virchows Arch 2015;466:245-254. 
3. Seethala RR, Stenman G. Update from the 4th edition of the World Health Organization Classification of Head and Neck Tumours: tumors of the salivary gland. Head Neck Pathol 2017;11:55-67.

4. Skálová A, Vanecek T, Majewska H, et al. Mammary analogue secretory carcinoma of salivary glands with high-grade transformation: report of 3 cases with the ETV6-NTRK3 gene fusion and analysis of TP53, $\beta$-catenin, EGFR, and CCND1 genes. Am J Surg Pathol 2014;38:23-33.

5. Solomon JP, Benayed R, Hechtman JF, et al. Identifying patients with NTRK fusion cancer. Ann Oncol 2019;30(Suppl 8):viii16-22.

6. Cocco E, Scaltriti M, Drilon A. NTRK fusion-positive cancers and TRK inhibitor therapy. Nat Rev Clin Oncol 2018;15:731-747.

7. Vaishnavi A, Le AT, Doebele RC. TRKing down an old oncogene in a new era of targeted therapy. Cancer Discov 2015;5:25-34.

8. Okamura R, Boichard A, Kato S, et al. Analysis of NTRK alterations in pan-cancer adult and pediatric malignancies: implications for NTRK-targeted therapeutics. JCO Precis Oncol 2018;2018:1-20.

9. Hong DS, DuBois SG, Kummar S, et al. Larotrectinib in patients with TRK fusion-positive solid tumours: a pooled analysis of three phase $1 / 2$ clinical trials. Lancet Oncol 2020;21:531-540.

10. Drilon A, Laetsch TW, Kummar S, et al. Efficacy of larotrectinib in TRK fusion-positive cancers in adults and children. N Engl J Med 2018;378: 731-739.

11. Doebele RC, Drilon A, Paz-Ares L, et al. Entrectinib in patients with advanced or metastatic NTRK fusion-positive solid tumours: integrated analysis of three phase 1-2 trials. Lancet Oncol 2020;21:271-282.

12. Hyman $D$, Kumar $S$, Farago $A$, et al. Phase I and expanded access experience of LOXO-195 (BAY 2731954), a selective next-generation TRK inhibitor (TRKi) [abstract]. Presented at American Association for Cancer Research Annual Meeting; March 29-April 3, 2019; Atlanta, Georgia. Abstract CT127.

13. Drilon $\mathrm{A}$, Siena $\mathrm{S}, \mathrm{Ou} \mathrm{SI}$, et al. Safety and antitumor activity of the multitargeted Pan-TRK, ROS1, and ALK inhibitor entrectinib: combined results from two phase I trials (ALKA-372-001 and STARTRK-1). Cancer Discov 2017;7:400-409

14. Drilon A, Li G, Dogan S, et al. What hides behind the MASC: clinical response and acquired resistance to entrectinib after ETV6-NTRK3 identification in a mammary analogue secretory carcinoma (MASC). Ann Oncol 2016;27:920-926.

15. Drilon $A E$, DuBois SG, Farago AF, et al. Activity of larotrectinib in TRK fusion cancer patients with brain metastases or primary central nervous system tumors [abstract]. J Clin Oncol 2019;37(Suppl):Abstract 2006.
16. Sasaki E, Masago K, Fujita S, et al. Salivary secretory carcinoma harboring a novel ALK fusion: expanding the molecular characterization of carcinomas beyond the ETV6 gene. Am J Surg Pathol 2020;44:962-969.

17. Drilon A, Oxnard G, Wirth $L$, et al. Registrational results of LIBRETTO001: a phase 1/2 trial of LOXO-292 in patients with RET fusion-positive lung cancers [abstract]. J Thorac Oncol 2019;14:S6-7. Abstract PL02.08.

18. Wirth LJ, Sherman E, Robinson B, et al. Efficacy of selpercatinib in RET-altered thyroid cancers. N Engl J Med 2020;383:825-835.

19. Drilon A, Oxnard GR, Tan DSW, et al. Efficacy of selpercatinib in RET fusion-positive non-small-cell lung cancer. N Engl J Med 2020;383:813-824.

20. Drilon $A$, Zhai $D$, Deng $W$, et al. Repotrectinib, a next generation TRK in hibitor, overcomes TRK resistance mutations including solvent front, gatekeeper and compound mutations [abstract]. Presented at the American Association for Cancer Research Annual Meeting; March 29-April 3, 2019; Atlanta, Georgia. Abstract 442.

21. Cocco E, Schram AM, Kulick A, et al. Resistance to TRK inhibition mediated by convergent MAPK pathway activation. Nat Med 2019;25:1422-1427.

22. Vaishnavi A, Scherzer MT, Kinsey CG, et al. Inhibition of MEK1/2 forestalls the onset of acquired resistance to entrectinib in multiple models of NTRK1-driven cancer. Cell Rep 2020;32:107994.

23. Hemming ML, Nathenson MJ, Lin J-R, et al. Response and mechanism of resistance to larotrectinib and selitrectinib in metastatic undifferentiated sarcoma harboring oncogenic fusion of NTRK1. JCO Precis Oncol 2020;4:79-90

24. Long GV, Flaherty KT, Stroyakovskiy D, et al. Dabrafenib plus trametinib versus dabrafenib monotherapy in patients with metastatic BRAF V600E/ K-mutant melanoma: long-term survival and safety analysis of a phase 3 study. Ann Oncol 2017;28:1631-1639.

25. Prahallad A, Sun C, Huang $S$, et al. Unresponsiveness of colon cancer to BRAF(V600E) inhibition through feedback activation of EGFR. Nature 2012;483:100-103

26. Kopetz S, Grothey A, Yaeger R, et al. Encorafenib, binimetinib, and cetuximab in BRAF V600E-mutated colorectal cancer. N Engl J Med 2019; 381:1632-1643.

27. Kopetz S, Grothey A, Van Cutsem E, et al. Encorafenib plus cetuximab with or without binimetinib for BRAF V600E metastatic colorectal cancer: updated survival results from a randomized, three-arm, phase III study versus choice of either irinotecan or FOLFIRI plus cetuximab (BEACON CRC) [abstract]. J Clin Oncol 2020;38(Suppl):Abstract 4001.

28. Sicklick JK, Kato $\mathrm{S}$, Okamura R, et al. Molecular profiling of cancer patients enables personalized combination therapy: the I-PREDICT study. Nat Med 2019;25:744-750 